\title{
Environmental Design for Patient Families in Intensive Care Units
}

\author{
Mahbub Rashid, PhD, RA \\ School of Architecture, Design, and Planning, University of Kansas \\ 1465 Jayhawk Boulevard, Lawrence, Kansas 66045, \\ E-mail:mrashid@ku.edu
}

\begin{abstract}
The purpose of this paper is to define the role of environmental design in improving family integration with patient care in Intensive Care Units (ICUs). It argues that it is necessary to understand family needs, experience and behavioral responses in ICUs to develop effective models for family integration. With its two components - the "healing culture" promoting effective relationships between caregivers and care seekers, and the "environmental design" supporting the healing culture-a "healing environment of care" can be an effective family integration model. This paper presents evidence showing how environmental design may affect families in ICUs, and proposes design recommendations for creating a healing environment of care promoting family integration in ICUs.
\end{abstract}

Keywords: intensive care unit (ICU), environmental design (ED), healing environment, healing culture, patient family

\section{INTRODUCTION: WHY FAMILY INTEGRATION WITH PATIENT CARE IS IMPORTANT IN ICUS}

The Society of Critical Care Medicine (SCCM) and the American College of Critical Care (ACCC) have adopted the definition of "family" provided in the Palliative Care Guidelines: "Family is defined by the patient or in the case of minors or those without decision making capacity by their surrogates. In this context, the family may be related or unrelated to the patient. They are individuals who provide support and with whom the patient has a significant relationship" [1]. Since critically ill patients are often unable to communicate and make decisions, families can make important contributions to care decisions on behalf of these patients [2-3]. Families can also help busy ICU nurses and physicians become more effective and efficient [4-5]. Simpson [6] studied various supportive functions of families in ICUs. Simpson's findings, as represented in Figure 1, fall well within the natural role of a family, as defined by Caplan [7].

To recognize the importance of families in patient care, the concept of familycentered care was developed [8]. Importance of the family was also recognized in the 


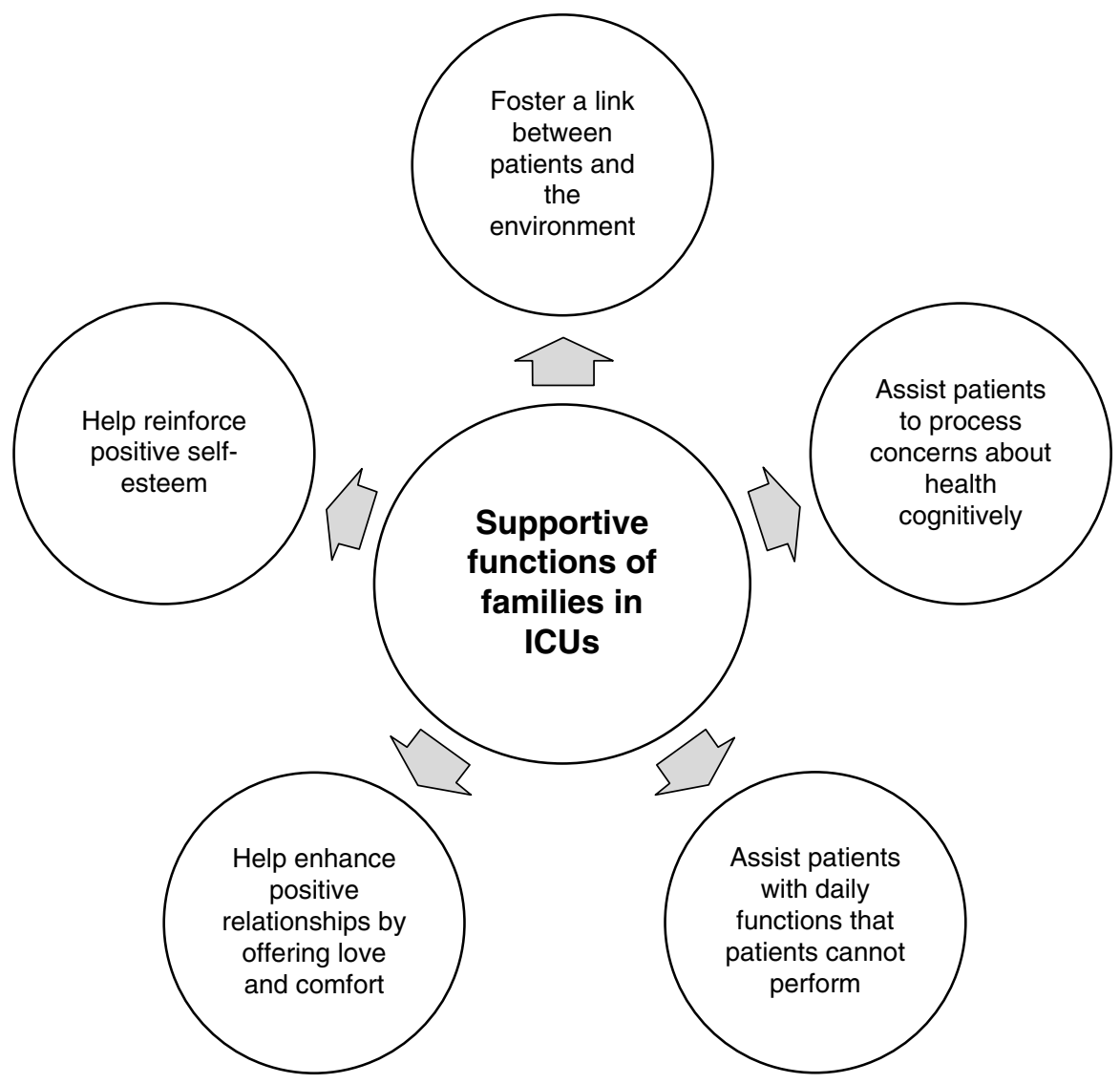

Figure 1. The supportive functions of families in ICUs based on Simpson [6].

patient-centered model of care [9]. The key elements of these models are quite similar: a) keep patients and families informed and actively involved in medical decisionmaking and self-management; b) coordinate and integrate patient care across groups of healthcare providers; c) provide the physical comfort and emotional support for patients and family members; d) understand patients' concepts of illness and their cultural beliefs; and e) understand and apply principles of disease prevention and behavioral change appropriate for diverse populations.

Several studies have demonstrated that patient-/family-centered care is associated with better clinical outcomes [10-13]. At the same time, patients and families are expressing a desire for a larger role in healthcare decision-making and are asking providers to do a better job of responding to patient and family needs [14-16]. Despite these concerns, families and other surrogates often feel uninformed, and disenfranchised from clinical decision-making and day-to-day care of loved ones in the ICU [17-18]. To 
achieve a culture of patient/family centered care aimed at integrating families with patient care, an environment to support the care model must be considered.

This paper introduces the concept of a healing environment of care as a model for family integration in ICUs, and argues that most existing models of care focus primarily on the cultural dimensions of healing and disregard the role of environmental design (ED) dimensions in promoting family integration. It presents related research evidence on the effects of ED on family needs and experience, and makes ED suggestions aimed at promoting family integration with patient care in ICUs. It also discusses barriers to implementing healing design in ICUs.

\section{METHODOLOGY}

An interpretive research methodology was adopted in reviewing the existing research evidence on the needs, experience and behavioral responses of patient families in ICUs. Also reviewed is the existing research evidence on ED issues concerning patient families in ICUs and healthcare facilities. The paper then presents the evidence as it informs the concept of a healing environment of care.

In order to identify potentially relevant articles published in English, three interrelated searches based on keywords were conducted. In the first search, needs, experience, and behavioral responses were used as keywords with patient families or relatives in ICUs. In the second search, various ED factors such as noise, music, lighting, color, artwork, air quality, smell, and nature were used as keywords with patient families or relatives in ICUs. In the third search, behavioral, social, and psychosocial issues such as control, privacy, communication, interaction, social support, wayfinding, religion, culture, and spirituality were used as keywords with patient families and ED in ICUs.

Cross-searches were conducted using combinations of keywords through the EBSCO research database. The database provided simultaneous access to Academic Search Premier, Alt Healthwatch, MED-LINE, PsycArticles, Psychology and Behavioral Sciences Collection, PsycINFO, and CINAHL. Additional searches were conducted through PubMed and Google Scholar. Further searches were conducted in the reference lists of a select set of recently published review articles to identify potentially relevant articles.

The title, abstract, and the date of publication of each potentially relevant article were reviewed. Articles were sorted in three groups based on their relevance to patient families, ED factors, and various behavioral and psychosocial factors in ICUs and other healthcare facilities. All articles selected for further review and analysis were published in peer-reviewed journals. In selecting these articles, no preference was observed in terms of research methodology. These articles reported studies using qualitative, quantitative, or mixed-methods research methodologies. However, all articles were evaluated in terms of the quality of research design and methods. Recently published articles on families in ICU, healing design, and patient-centered care were given additional emphasis in the process of review and analysis. Some recently published articles on ED factors in office settings were also included in the review, because 
environmental effects on healthy individuals in office settings may be somewhat similar to that on patient family members in healthcare settings.

Limitations to the methodology pertain primarily to ED findings and recommendations concerning patient families. Very few studies have reported effects of ED on families in ICUs. Even fewer studies have reported studies that focus on design interventions in ICUs aimed at improving family outcomes. Altogether, only 10 out of all the cited articles in this paper are directly relevant to ED for patient families in ICUs [19-28].

\section{FAMILY NEEDS, EXPERIENCE AND BEHAVIORAL RESPONSES IN ICUS}

As a first step toward integrating families with patient care in ICUs, many studies have focused on the needs and satisfaction of family members during ICU stays [5, 29-42]. In an important study to determine the perceived needs of families of critically ill patients, Molter [5] developed a list of 45 need statements to be rated on a scale of 1 to 4. In the study, Molter found that many of the most important needs identified corresponded well to the comfort measures identified in other studies. Further, none of the 10 most important needs identified by her subjects was influenced by age and socioeconomic status, indicating that these needs are very important to most family members and, therefore, should be a major concern to caregivers. Molter and Leske [36] revised Molter's initial list to create the Critical Care Family Needs Inventory (CCFNI) for family assessment and self-report of specific needs. Daley [32], Norris and Grove [30], Rodgers [32], and others replicated Molter's work in other critical care settings with comparable findings. Leske [37] studied the psychometric properties of the tool, and identified five categories of needs: assurance, proximity, information, comfort, and support needs (Figure 2). Other studies reconfirmed the importance of Leske's family needs categories [43-49]. Therefore, the importance of designing an ICU to meet these categories of needs has been established.

Other related lines of inquiry showed that needs are viewed differently by family members and health care professionals $[38,40,50]$. Thus, nurses often provide routine responses and generalized interventions to family members and waste energy attempting to respond to nonexistent needs. Norris and Grove [40] found that nurses tended to underestimate the needs of family members. Lynn-McHale and Bellinger [38] found that among the six categories based on the needs statements of Molter's instrument, personal support systems, visitation, information, psychological aspects significant to the family, environmental factors, and institutional support systems, it was only the last category on which nurses and family members agreed in their estimates of satisfaction. These findings suggest that caregivers and family members have different priorities concerning ICUs. While ICUs are often designed to meet the priorities of caregivers, it is important to conduct a gap analysis between caregivers' and families' priorities in designing ICUs.

In addition to many studies on needs and satisfaction levels of family members of critically ill patients, both quantitative and qualitative studies on the experience of family members - their emotional distress, and affective and behavioral responses - have also been reported [20, 21, 29, 47, 51-59]. Kleiber and colleagues [47] asked family 


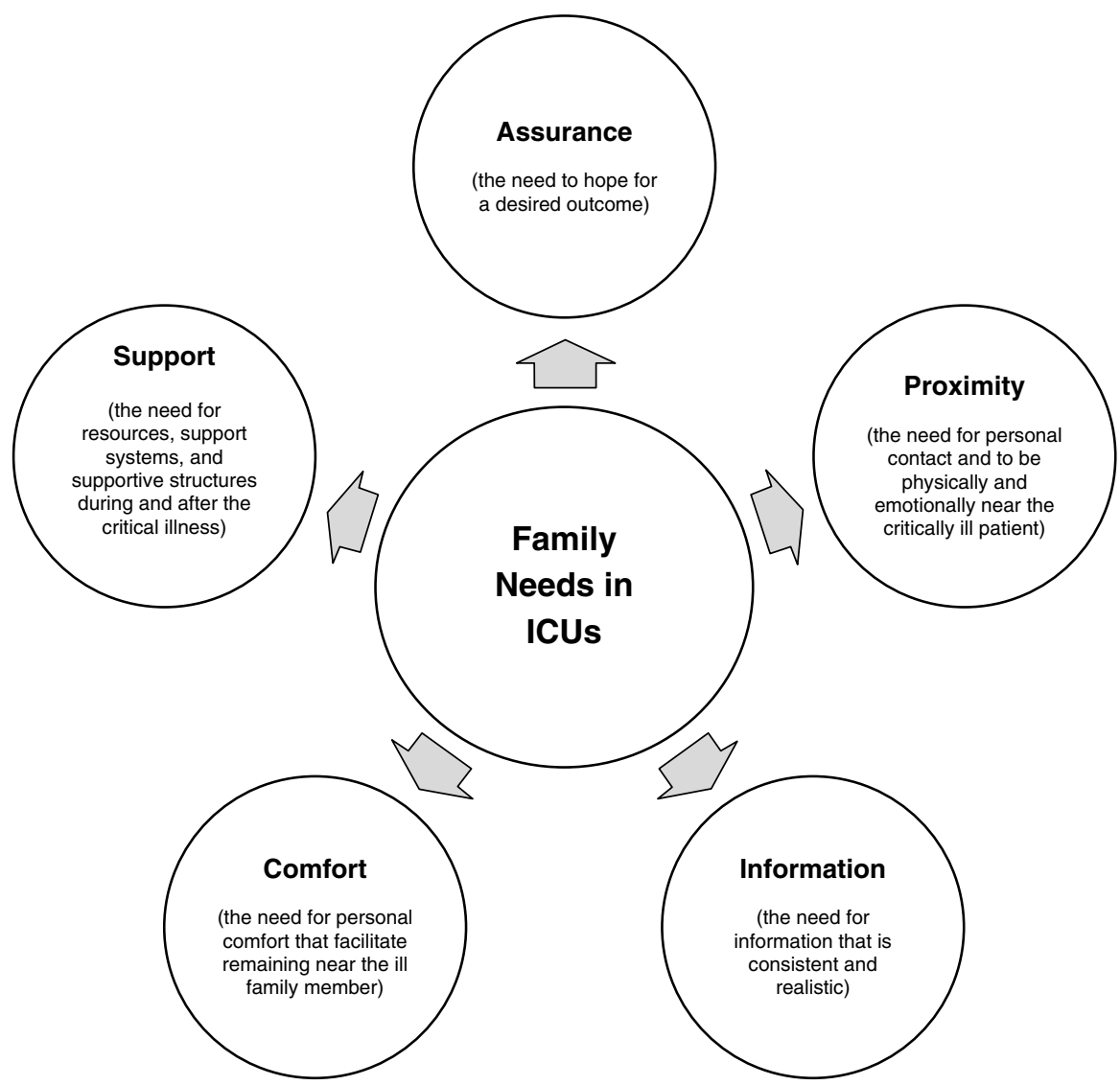

Figure 2. Leske's five categories of family needs in ICUs [37].

members in different ICUs in a large tertiary care hospital to keep daily log of their experience and feelings. Thematic analysis of the log revealed a broad range of powerful emotions, including sadness, depression, hate, anger, exhaustion, and fatigue. Appreciation for the support offered by others was expressed frequently. A commonly identified supportive behavior was providing information about the patient's condition. Quiet presence was apparently viewed positively by many family members. Communication, which was considered different from providing information, was described as sharing of thoughts and feelings through talking to and listening to friends and relatives. Other supportive behaviors were assistance and comfort. The study concluded that based on families' emotional responses, it is necessary to support the families throughout the ICU stay, and indicates a need for flexible family spaces designed to promote comfort, social interaction, and privacy. 
In a more recent qualitative study directly addressing the environment, Kutash and Northrop [20] described family experiences of waiting rooms in adult ICUs using semistructured interviews. Six categories of themes emerged from data analysis revealing both structural and subjective aspects of waiting:

1. Close proximity referred to the importance of a close physical distance to patients, which was central to easing the waiting period. Regarding close proximity, Bournes and Mitchell [21] found that family members talked about "not wanting to go too far away - just in case", and "being there to see that he is still breathing".

2. Caring staff captured the comfort family members felt when staff showed caring behaviors towards loved ones. However, family members did not focus on caring behaviors directed towards them.

3. Need for a comfortable environment represented the impact of the design of the waiting room on family well-being. The study found that family's perceptions of the waiting room were mostly negative. Some described the room/space as cold, dirty, a small space and "a place to go, not to stay". Others suggested that the waiting room needed to be a little bit more human friendly. Yet others described the furniture as uncomfortable to sit in and too close together. They talked about having water in the cooler and having enough clean blankets and pillows.

4. Emotional support referred to the fact that family members found the waiting room to be a place where they could obtain emotional support. It was described as a place where "one family consoles another family" and "a place where you can be with people in similar situations". Rodgers [42], who observed behaviors and interactions of individuals who shared an ICU waiting room, described it as a community of "former strangers who had become united by some common experience".

5. Rollercoaster of emotions captured the range of emotions experienced by family members.

6. Information referred to the importance of receiving information about their loved ones. The comments by participants in this study suggested that the waiting room enhances the ability of family members to receive information about their loved ones by face-to-face communication.

These findings indicate a need to design waiting spaces to promote a comfortable and calming environment, and to give families easy access to and sufficient visibility of patients. They also indicate a need to design waiting spaces to enhance communication between the healthcare team and families, and to facilitate better access to information.

In an interesting study, Burr [58] used both CCFNI and interviews to provide a contextual representation of family needs in ICUs. Her results indicated at least two major needs that are not included in CCFNI: the need of family members to provide reassurance and support to the patient, and their need to protect patient and family. The family members' need to support and reassure the patient was a common response to the feelings of helplessness. The need to protect was revealed through concerns to prevent or minimize anything that would upset or worry the patient. Information was withheld if it was considered disturbing to the patient. Other visitors were screened for the same reason. The need was also related to preserving the patient's dignity and privacy. These findings indicate a need for designing an ICU to include space to sit vigil and to promote patient privacy and dignity. 
In addition to the quantitative studies of family needs and the qualitative thematic studies of family's experience, several quantitative studies on family members' behavioral responses related to ICU experience are also reported in the literature. In one quantitative study, Halm and colleagues [49] found that family members slept less, had poorer sleep, took fewer naps, and slept in the waiting room about one third of the time. They changed eating behaviors, and ate more snacks and "junk" food to limit the amount of time they were away from the ICU. They spent less time watching television and more time talking, visiting the patient, and waiting during the critical illness. The majority of family members reported feeling "very tired". Finally, they all had a very high level of stress at the time of the critical care admission. Stress began to plateau on Day 6 and then dropped considerably by Day 28. In other words, over time, most family members successfully adapted to the stress associated with critical care hospitalization, a finding that was confirmed by Auerbach and colleagues [59]. These behavioral responses indicate that family spaces in ICUs need to be quiet and calming to promote rest and sleep. They also need to be conducive to social interaction. Probably, it would be best for families to get food within the unit so that they can always be close to patients. Finally, the environment needs to therapeutic and free of stressors of any form.

In summary, studies on families in ICUs suggest that their needs, experience, and affective responses are important from the viewpoint of patient-/family-centered care. These studies also indicate several ICU design issues concerning these needs, experience, and affective responses. A discussion of these design issues within the framework of the healing environment of care is in order.

\section{THE HEALING ENVIRONMENT OF CARE AS A MODEL FOR FAMILY INTEGRATION}

In a study conducted by the Picker Institute [60], patients and families disclosed or related that they considered healthcare environments to be "healing" when care was attentive to mind, body, and spirit, when they felt they had a relationship with their healer, and when they were actively involved in the decision-making process. Fundamentally, a deliberate caring relationship between a caregiver and the patient and patient's family is the core of the healing environment. In general, a healing environment can be viewed as having two components - the intangible culture of care and the tangible healing design.

\subsection{The healing culture of care}

A healing culture of care is that in which there is an obvious regard for the dignity of human beings, and where relationships between care seekers and caregivers are built on mutual respect and a shared commitment to healing. In such a culture, caregivers understand that each encounter with patients and their families is an opportunity to convey care and respect-from gathering and giving information at the time of admission, to responding to a request, to transporting patients, to stopping to offer directions in the hallway, or to simply acknowledging someone who is in need. This means that every member of the hospital staff, regardless of their job description, has a role to play in a healing culture. 
Many hospitals and ICUs have proposed models/programs for improving their culture of care. They focus primarily on behavioral and organizational changes including changes in (a) visitation policies (b) decision-making processes regarding patient care, (c) medical round practices, (d) cultural, religious and spiritual support, and (e) in staff interaction with family members. Without an environment to support family presence, these mandates could increase staff stress.

Dowling and her colleagues [61] reported that family satisfaction ratings were impacted more favorably by the family's perception of their feeling safe and secure in the hospital environment than by the family's perception of the culture of communication and care in ICUs. The same study also reported environmental amenities such as "a private place in the hospital to sleep and rest while waiting" and information related to the environment, such as "information on local places to stay or eat" having a positive impact on family satisfaction ratings [61]. Therefore, in addition to the culture of care it is necessary to consider the ED dimensions of ICUs in relation to family perception, experience, and behavioral responses if the goal is to improve family integration with patient care in ICUs.

\subsection{The concept of healing design}

Unlike the mostly intangible healing culture, a tangible healing design makes healing intentions evident in the way it attends to the entire experience of its users. The healing design needs to be comforting and pleasant to all human senses. For example, an overemphasis on visual needs may undermine other sensual needs of users. However, it is not enough for a healing design to be pleasant to human senses only. It must also be uplifting, inspirational, and even spiritual. Additionally, a healing design needs to be sensitive to the socio-cultural needs of its users. It recognizes that what works well for one may not work well for another. Regarding healthcare settings, the healing design must not contradict the culture of care. For example, an unrestricted visiting policy in an ICU may become ineffective or counterproductive if there is not enough family space in the unit or patient room; in a shared decision-making culture, it may be difficult to organize meetings between staff and family as needed if meeting spaces are not available. In sum, the healing design involves the physiological, psychological, as well as socio-cultural wellbeing of its users.

So far, the role of the healing design in family integration models/programs has remained marginal. This is in part due to a hospital organization that is economically challenged, and in part due to the ineffective role of architects/designers in raising ED awareness and in creating healing environments where ED provides significant restorative benefits. Most hospital administrators emphasize processes related to efficiency, often causing hospitals to become noisy, cluttered, unpleasant environments with little regard for the potentially detrimental effects of these environments on the physical and/or psychological well-being of patients, families, and staff. Only recently, do we find some hospitals beginning to invest in ED for improving the experience of patients and their families. The reasons for this change can be found in the environmental research design literature. 


\title{
5. ENVIRONMENTAL DESIGN FOR PROMOTING FAMILY INTEGRATION IN ICUS
}

\author{
5.1. Environmental design recommendations for family waiting areas/spaces: \\ literature review
}

Though studies on the effects of ED on family needs, experience, and behavioral responses in healthcare settings in general and in ICUs in particular are rare, the effects of ED on patients and staff are somewhat well studied. As more ICUs seek to integrate families with patient care, families are likely to spend more time with their loved ones in ICUs. Consequently, families may be affected by some of the same ED features affecting patients and staff in ICUs, though not always to the same extent. Many excellent review articles report the effects of these environmental features on patient, family/visitor, and staff outcomes at some length [19-26, 62-89]. These review articles look at studies involving almost every environmental design feature including noise, music, lighting, aroma, air quality, and view of and access to nature. They also look at studies involving different behavioral, social, psychosocial and spiritual factors.

Most comprehensive among the recent review articles are the two by Ulrich and his colleagues [84-85]. Ulrich et al. [84] review more than 600 peer-reviewed articles published since 1968 on the effects of physical environment on healthcare outcomes. The other review [85] includes those studies published between 2004 and 2008, and those that report findings in areas of healthcare environment not included in their previous article. Rashid \& Zimring [78] review about 250 articles reporting the effects of indoor environment in healthcare and office settings. This review article is relevant to this study because environmental effects on healthy individuals in office settings may be somewhat similar to that on patient family members in healthcare settings. Other reviews on healing design can be found in [19, 23, 24, 25, 26, 80, 89].

Table 1 presents a summary of the environmental design research findings for different important environmental issues, and Table 2 presents the same for behavioral, social, and psychosocial issues. The tables include only the findings that appear relevant to families in ICUs. They also contain ED recommendations in relation to the findings. The recommendations are quite generic in nature, and therefore can be applied to any hospital spaces to be used by patient families during the course of their stay in the hospital. Specific ED recommendations for family areas, that are not included in the tables, are discussed below.

\subsection{Environmental design recommendations for family waiting areas/spaces: the author's perspective}

The location of family waiting spaces in ICUs is very important. It defines visual and physical access of families to patients in ICUs. It also signals the degree to which families are integrated with patient care [27]. Family spaces can be provided outside the unit, inside the unit, within the patient room, and/or at more than one of these locations. Family spaces located outside the unit may suggest that families are not integrated with patient care in the unit. On the other hand, family spaces provided within the patient room may indicate that families are well integrated with patient care. Family spaces provided within the unit but not in the patient room may indicate that the role of the 


\section{Table 1. Research findings and related ED recommendations on various environmental issues relevant to patient families in ICUs}

Noise [23, 63, 65, 78, 84-86, 88, 90-101]

- Hospitals are very noisy places, with noise levels far exceeding the WHO guidelines.

- Sources of noise in hospitals are numerous, and can easily be eliminated.

- Noise is a major source of sleep disruption, hence discomfort, in healthcare settings.

- Noise can have significant effects on annoyance and stress.

- People are more comfortable if they can control noise when needed.

- Hospital floors, ceilings, and walls are typically hard surfaces that reflect sound and thus contribute greatly to noise.

Music [63, 68, 71, 87, 102-107]

- Relaxing music or therapeutic sounds can provide positive distraction and promote the healing process.

- Music releases tension, decreases pain, and help a person focus on awareness.

- Music is a proven intervention for anxiety in ICUs.
- Include carpeting in corridors to lessen the impact of footsteps, rolling carts, and staff member conversations.

- Locate storage areas, staff lounges, and utility rooms away from patient rooms and family spaces.

- Locate internal corridors between storage and utility rooms allowing clinical and support staff to conduct necessary tasks without disturbing patients and families.

- High-performance sound absorbing materials can be used to reduce reverberation time, sound propagation, and noise intensity levels, as well as to improve sleep.

- Use music to mask distressing environmental noise that cannot otherwise be eliminated.

- Sounds of nature accompanied by soft classical music can be used in family waiting areas to calm anxious family members or visitors.

- Consider the following when providing music in family areas:

- Not all music can produce a desired calming effect.

- Music often evokes emotions and feelings that are rooted in an individual's past experiences and personal preferences.

○ It is essential to give families a sense of control regarding their music preferences. 
Table 1. Research findings and related ED recommendations on various environmental issues relevant to patient families in ICUs (Continued)

Lighting [69, 78, 84-86]

- Lighting, natural and artificial, can have both positive and negative effects on humans.

- The effects of lighting can be visual, emotional, or biological in nature.

- The effects of light relate not only to the intensity and duration of exposure but also to the pattern of lighting.

- Appropriate lighting can help uplift one's mood. It can also create a relaxed ambience.

- Interruptions in normal light-dark patterns may disrupt normal physiological processes.

- Continuous lighting and the absence of natural light may cause cognitive disturbances.

- The ratio of hours of light versus hours of darkness may influence circadian rhythms of humans.

- Higher color temperature or higher light levels increase alertness.

- Any affective response to lighting are often determined by traumatic experiences or by such factors as education, and social and cultural values.

- Low-level lighting seems to encourage longer conversations.

- People feel uncomfortable if they are unable to control lighting levels to suit their own requirements.

- Some negative effects of fluorescent light with magnetic ballasts can be solved using digital ballasts.

- Full-spectrum fluorescent lighting has some of the same emotional and physiological effects as natural sunlight.
- Consider the following regarding the visual, emotional, and biological effects of lighting:

- The right balance of lighting is often a function of the space and the activities in the space.

- In general, the biological and visual aspects are important in functional areas such as nurse stations.

- In family spaces, the emotional aspect may play an important role.

○ For patient rooms, lighting must be accommodated in all three aspects in some combination.

- Consider the following regarding natural light in family spaces:

- Attention should be given to making natural light available in all family spaces in the unit.

- If the family space is within the patient room, make sure that the intensity of natural light is comfortable to patients.

- The use of slightly tinted or reflective glass can effectively reduce glare and heat production from natural sunlight.

- Vertical blinds and other window treatments can be used for adjusting light intensity as desired by the patient.

- Consider the following regarding artificial light in family spaces:

○ It is important to consider multiple options for lighting that can be controlled when desired.

- Where natural light is not an option (older ICUs), consider using fullspectrum fluorescent lighting.

- A dynamic lighting solution that allows the color and temperature levels 
Table 1. Research findings and related ED recommendations on various environmental issues relevant to patient families in ICUs (Continued)

ED Recommendations

to be changed according to the time of day may be suitable for a patient room or a family space.

- Consider the following regarding lighting in patient rooms:

$\circ$ The intensity and direction of all light sources should be controllable.

○ A softened lighting option should be planned in addition to the technical lighting by installing wall sconces or lights on dimmers to provide a more relaxing atmosphere for the patient and family.

- Night lighting must also be considered since the patient will be frequently checked and observed by the family or the clinical staff.

○ While providing ambient light, glare needs to be prevented from interfering with monitoring screens.

- Avoid heat gain from ambient light sources on sensitive oximetry and ventilator alarm systems.

In sum, when selecting lighting options in critical care units, a variety of characteristics must be considered: technical lighting needs, soft lighting for relaxing, and night lighting, as well as the location, intensity, and controllability of the lighting. Any lighting solution that lacks variability and controllability may fail to meet individual demands in the stressful environment of ICUs. Also, light switches need to be placed ergonomically and in a location accessible by the family without interfering with equipment. 
Table 1. Research findings and related ED recommendations on various environmental issues relevant to patient families in ICUs (Continued)

Findings

ED Recommendations

Color and Artwork [75, 84-86, 108]

- Colors affect emotion and physiological responses.

- Blue, green, and violet have calming and restful effects, while pastels provide a more cheerful quality than do deeper shades.

- Soft and mixed tones that do not have sharp contrasts promote rest.

- Art representing nature evokes positive response and abstract art evokes negative response.

- Emotionally inappropriate art subject matter or styles can increase stress and worsen other outcomes.

- Artwork and color can easily help patients and families who are confused and need diversion.

- Consider the following in providing color and artwork in ICUs:

o Color and artwork in ICUs should be calming.

- Patients and families in ICUs have different culture and religion. Thus, in selecting artwork, designers need to be sensitive to culture, religion, and the specific geographical area.

o Develop systems involving artwork that can be changed at will by the patient and family as easily as changing channels on a television.

○ In rooms that do not have the benefit of an outside window, a sunny, light colored, bright picture can provide a sense of nature and light.

○ Art varies enormously in subject matter and style. Therefore, it may be unreasonable to expect all art to be suitable for high stress healthcare spaces.

Smell [67, 79, 84-86]

- Pleasing aromas can reduce blood pressure, slow the rate of respiration, lower pain-perception levels, improve the immune system, and help increase a sense of well-being.

- Jasmine and rosemary increase beta waves, thus increasing alertness. Likewise, lavender increases alpha waves, thereby promoting relaxation.

- Fragrance perceived as moderately to extremely pleasant may help lower anxiety.

- Odors ("negative smells") may stimulate anxiety, fear, and stress.

- Consider the following regarding the use of aroma in ICUs:

- Hospitals, particularly ICUs, are well known for their unpleasant odors or chemical smells.

- Smell is acutely retained in memory (even more than sound or visual images).

- Strong-smelling cleaning agents should be avoided near patients and families.

- Aroma should be used sensitively to promote a healing environment for patients, families, and staff. 
Table 1. Research findings and related ED recommendations on various environmental issues relevant to patient families in ICUs (Continued)

ED Recommendations

Air Quality [78, 84-86]

- Low ventilation rates (less than 10 liters/ second per person) in all building types may be associated with statistically significant worsening in one or more health or perceived air quality outcomes.

- Increased ventilation rate may be associated with a significant decrease in the prevalence of Sick Building Syndrome (SBS) symptoms or with a significant improvement in perceived air quality.

- The risk of SBS symptoms may decrease significantly with carbon dioxide concentration below 800 parts per million.

- Low air supply velocity is generally more comfortable.

- Hospital infection rates are affected by the types of air filter, directions of airflow, air pressure, air change rates, humidity, and ventilation system cleaning and maintenance regimes.

- Hospital construction and renovation activities are related to airborne infection outbreaks.

- Patient families may be at risk of contracting infectious diseases due to airborne contamination.

Nature [72-4, 82-86]

Viewing Nature

- Positive feelings such as pleasantness and calm increase, and anxiety, anger, or other negative emotions diminish because of exposure to nature.

- Many nature scenes are able to sustain positive interest, and thus function as pleasant distractions that may block worrisome, stressful thoughts.
- Consider the following to improve air quality in ICUs:

- Install effective high efficiency particulate air (HEPA) filters.

- Employ appropriate ventilation systems and air change rates.

- Employ appropriate control measures to contain airborne contamination during construction or renovation.

- Use single-bed rooms instead of multi-bed rooms to increase isolation capacity and reduce transmission from infected patients.
- Because of the positive effects of nature on physical and emotional wellbeing, it is preferable to design family areas with windows to the outside.

- If possible, these spaces need to be close to hospital gardens with plants, water, and other natural objects. 
Table 1. Research findings and related ED recommendations on various environmental issues relevant to patient families in ICUs (Continued)

- Viewing built environments that lack nature (e.g., parking lots, rooftops, and rooms without trees and/or plants) have less restorative effects and may even increase stress.

- Prolonged visual contact with nature may have persistent positive effects on psychological, physiological, and possibly behavioral components of stress.
- When family spaces do not have view of or access to nature, consider bringing nature within the unit. Along with potted plants and sound of nature, use nature related artwork for the purpose.

- Consider adding paintings or murals of nature scenes on a wall, if the views out of windows are without the opportunity for actual views of nature.

\section{Experiencing Nature}

- Being in gardens can be restorative.

- Gardens can reduce stress through other mechanisms, such as fostering access to social support, restorative escape, and control with respect to stressful clinical environments.

- Patients, families, and staff who use hospital gardens report reduced stress and improved emotional wellbeing.

- Gardens tend to alleviate stress more effectively when they contain green or verdant foliage, flowers, water, grassy spaces with trees or large shrubs, a modicum of spatial openness, and compatible pleasant nature sounds, such as birds and water.

Table 2. Research findings and related ED recommendations on various behavioral, social, and psychosocial issues relevant to patient families in ICUs

ED Recommendations

Sense of control $[65,109-11]$

- Humans, in general, have a strong need for control-a concept that subsumes many other psychosocial concepts including privacy.
- Families who can control the temperature and lighting in their rooms, the amount of privacy they have, the number, frequency and length of visitation,

(Continued) 


\section{Table 2. Research findings and related ED recommendations on various behavioral, social, and psychosocial issues relevant to patient families in ICUs (Continued)}

Findings

- Uncontrollable situations or conditions usually are aversive and stressful.

- Having a sense of control with respect to a potential stressor help reduce the negative effects of the stressor.

- In hospitals and ICUs, patient families are under stress because they are unable to control noise; they have difficulties finding their ways; they have no personal control over lighting and temperature; and they frequently have no privacy.

Privacy [112-113]

- Privacy can be interpreted as the need to control or regulate access to the self. It is an important psychosocial need for humans.

- Privacy is often an important determinant of individual satisfaction.

- Despite the growing importance of privacy in healthcare settings, existing research in this area is sparse.

- More recently, the Health Insurance Portability and Accountability Act (HIPAA) provides additional reasons for redesigning patient care areas to enhance control and privacy.

- With increased family access to ICUs through open visiting, privacy becomes a family issue.

- Visual privacy and informational privacy including speech privacy are important in ICUs.

- Hard-wall partitions provide substantially better protection for speech privacy than curtains.
ED Recommendations

the type and volume of music, and the timing and content of meals will experience less stress and will likely be more satisfied.

- Giving patients and their families an opportunity to personalize their environments may also give them a sense of control and familiarity.

- Consider flexible furniture arrangements, or allow families to change furniture layout to meet their needs. Seating arrangements that cannot be changed or chairs that cannot be moved may cause frustration among families.

- It is necessary to express clearly in design the fact that privacy varies along a continuum ranging from the public to the intimate. A good ED allows a gradual transition between these extremes. Consider the following in this regard:

- A hospital lobby, the most public space of all hospital spaces, should be an open, welcoming area, yet not as open or as busy as the street outside.

- Public hallways leading to private patient areas, such as ICUs, should not be quite as open as a lobby, nor should they feel private.

- A tight public corridor in a hospital is a pervasive and intimidating major shortcoming.

- A generously wide public corridor may need to be much wider than the minimum dimensions often specified in design guidelines. 
Table 2. Research findings and related ED recommendations on various behavioral, social, and psychosocial issues relevant to patient families in ICUs (Continued)

- To enhance architectural privacy, consider the following:

- Use private patient rooms.

○ Use high-performance sound-absorbing materials.

- Use hard-wall partitions instead of curtain to protect speech privacy.

- Provide private discussion rooms to help reduce privacy breaches.

- Use wireless communication system to help eliminate the need to raise voices across large spaces or down hallways.

Communication, interactions, and social support [114-127]

- Staff-family communication can provide social support including emotional, informational, and tangible supports to family members, and can facilitate family members' involvement in patient care.

- Research on how ED enhances or hinders communication in healthcare settings is inadequate given the importance of the subject matter.

- Private patient rooms increase family and social support, as compared with multi-bed rooms. A clear advantage of private patient rooms in fostering social support stems from the fact that they provide more space and furniture for family and visitors.

- Decentralized registered nurses may be able to spend more time in patient rooms and less time waiting in medication and supply rooms. They may also visit patient rooms more often, creating opportunities for additional interactions, monitoring, and care.

- Furniture arrangements affect group behaviors including social interaction.
- Consider the following to increase opportunities for family-staff interactions:

- Centrally located nursing stations may limit family access to staff.

- Glass partitions around staff may limit family access to staff.

- Decentralized nursing stations may help create more opportunities for nurses to spend more time in patient rooms and to visit patient rooms more frequently for additional interactions, monitoring, and care.

o Private, rather than semiprivate, patient rooms as well as adequate consultation rooms, provide more opportunities for confidential discussions.

- Within hallways, alcoves can provide private spaces for confidential discussions.

- Arranging seating side-by-side along room walls inhibits social interaction.

- Private and peaceful spaces may help improve communication.

- People may interact longer in dim lighting conditions than in bright lighting conditions. 


\section{Table 2. Research findings and related ED recommendations on various behavioral, social, and psychosocial issues relevant to patient families in ICUs (Continued)}

- Softer flooring materials in patient rooms may make family members stay longer.

Wayfinding [62, 64, 128-135]

- As hospitals become more complex, poor wayfinding systems can be extremely costly in terms of both patient and family satisfaction and hard dollars.

- People tend to move along routes that are more accessible from a greater number of spaces; this emphasizes the importance of the overall structure of an environment from the viewpoint of wayfinding.

- You-are-here maps, directories, and signage are important wayfinding aids.

- When you-are-here maps are aligned in directions other than the forward position, people take much longer to find their destination and their efforts become significantly less accurate.

- People with necessary functional information upon reaching hospitals are more self-reliant and make fewer demands on staff. In contrast, people without the information rate the hospital less favorably and have elevated heart rates.

- People in hospitals who have access to more signs along the way are faster, less hesitant, ask for directions fewer times, and report lower levels of stress.

- In many complex facilities, first time visitors must spend extra time and energy to find their way around a poorly designed building.
- Wayfinding problems cannot be tackled piecemeal. The ED of a well-integrated wayfinding system should include external building cues, functional information, and an intelligible building layout.

- Wayfinding must be made a part of the building design process. Every time the building is renovated or changed, or departments change their locations or functions, wayfinding needs to be evaluated for appropriateness.

- It is important to identify routes that are, on average, more accessible from a greater number of spaces in the layout when placing important facilities and key points such as the entrance.

- Wayfinding must be considered keeping in mind that families with critically ill patients in ICUs often develop symptoms of anxiety, depression, and posttraumatic stress syndrome. As a result, they are more susceptible to information overload, less able to process information provided by the environment, and are more dependent on the assistance of others.

- Cafeterias or food courts needs to be easily accessible from family waiting areas, and appropriate directional maps and signs should be provided.

- Patient families may also be the first-time visitors to the hospital. Consider the following in this regard: 
Table 2. Research findings and related ED recommendations on various behavioral, social, and psychosocial issues relevant to patient families in ICUs (Continued)

- External signs and cues that lead to the hospital, especially from the parking lot, must be considered carefully because they are the first point of contact with the hospital.

- Consider numbering the floors on elevators in ways that help eliminate confusion.

- Directional signs should be placed at or before every major intersection, and at major destinations. Consider the following regarding signs:

If there are no key decision points along a route, signs should be placed at a regular interval.

Signs should have symbols that are easily understandable by all.

Signs should be in areas with sufficient lighting.

- Use such artifacts as sculptures, photographs, or paintings as important orientation cues.

- Atriums and galleries, instead of a long corridor, are effective wayfinding tools because of their distinctive design.

- A view of the sky, the outdoor, and/or the ground may help maintain orientation for visitors not yet familiar with the environment.

- Unique floor tiles, room carpet of a different color from the hallway, private patient mailboxes at the entrance of a room, and unique patient room entrances can help family members locate a specific room along a corridor of rooms. 
Table 2. Research findings and related ED recommendations on various behavioral, social, and psychosocial issues relevant to patient families in ICUs (Continued)

Findings

ED Recommendations

Religion, Culture, Spirituality [70, 71, 76, 81, 89, 108, 136-140]

- Culture and religion affect how people make sense of and respond to illnesses.

For some patients and families, illness is not only a physical and emotional crisis, but a spiritual calamity.

- A misunderstanding of cultural and religious differences among patients and families may become a barrier to optimal healthcare.

- Prayer and religious affiliation may be associated with health benefits.

- Most hospitals today are designed to meet the needs of technology rather than spiritual needs of patients, families, or staff.
- In designing ICUs, it is necessary for designers and care providers working with designers to have a working knowledge of common cultural and religious needs of patients and families.

- Focus groups with local spiritual and ethnic leaders may help identify most common design concerns of these groups.

- Nature may be the most important dimension of spirituality. In this regard, consider the following:

- Outdoor labyrinths in gardens can be a focus for spirituality.

- Nature may be useful in providing images of lifecycles such as birth, death, and the renewal of life.

- It may help families to have a chapel or a sanctuary close to the ICU where religious services can take place. Sometimes, these places are the only quiet refuge for families from the chaos of the hospital.

- In lieu of a chapel, a meditation room or an office of the chaplain near the unit may also work.

- Consider having space for religious books, inspirational texts, and texts on grief and coping written in multiple languages in the chapel or the community room.

- Consider having space for common religious items used by people of different faiths (e.g., rosaries, crucifixes, and holy water for Catholic users; clean clothing, prayer rugs and compasses for Muslim users; Sabbath kits for Jewish users; etc.) in the chapel or the community room. 
Table 2. Research findings and related ED recommendations on various behavioral, social, and psychosocial issues relevant to patient families in ICUs (Continued)

\begin{tabular}{|c|c|}
\hline Findings & ED Recommendations \\
\hline & $\begin{array}{l}\text { - When building a new unit, a tile marker } \\
\text { could be inserted in all family spaces and } \\
\text { patient rooms signifying the direction of } \\
\text { prayer. } \\
\text { - Consider providing transportation of the } \\
\text { hearse directly to the morgue in order to } \\
\text { protect privacy. } \\
\text { - Consider providing community rooms for } \\
\text { tai chi, yoga, and other spiritual } \\
\text { modalities to promote healing. }\end{array}$ \\
\hline
\end{tabular}

family in patient care is in a state of flux in the unit. Of course, such a reading of patient/family centered care based on spatial layout may at times be wrong. Some units may still not allow families to play an active role in patient care even with families present in the area designated for them in the patient room.

For families to feel safe and assured, they need access to both patients and their caregivers. Families are in distress when access is denied or restricted either to patients or to caregivers. Regardless of locations, family spaces should provide easy visual and/or physical access to the patient rooms because families would often need to see the patient. Families should also have easy access to caregivers when needed. It is generally accepted that, for patient safety it is best for families to enter the unit through a separate entry other than the one/s used by service and clinical staff. Designers must make sure that in designing such a system of entrances they do not make interfaces among families and caregivers difficult. Families must know when caregivers are available in the unit to answer their questions. If the ICU design restricts such interfaces, families may simply gather themselves at places where they are likely to find caregivers.

In laying out the family waiting area, the metaphor of home may be apt. Like home, waiting areas may be broken down into zones with varying degrees of privacy and control. Waiting areas should be large enough to accommodate all family members comfortably. Waiting areas can be sectioned to provide more intimate and quieter resting spaces and relatively busy and noisy activity spaces. Families with children should be given spaces separate from a "quite zone" for adults only. A play area for children within the direct visual reach of the adult family members should be considered in the area designated for families with children. Each section can be separated by solid partitions, dividers, glass walls, or planters depending on the need of these spaces. Each section should contain comfortable chairs and/or sofas for the 
family. Both resting and activity spaces should include private spaces or booths for telephone conversations. Additionally, activity spaces should not only include computers with internet access, but also allow families to access these computers to keep themselves up-to-date with patient status as well as with the outside world. Activity spaces should also include study carrels with health care information that families can access during their waiting periods. It is desirable to create a media room to separate the television from the rest of the waiting area, ensuring that families who wish to have quiet and solitude while waiting are not disturbed by television noise. Some hospitals are providing family sleep rooms with private bathrooms, kitchenettes and laundry in their waiting areas for family members who must stay at the hospital for an extended period of time. Such accommodations are helpful for family members who live a significant distance away from the hospital or who wish to stay with the patient.

Attention must be paid to furnishings in the family areas. Furnishings should be durable, comfortable, and cheerful. Furnishings should include comfortable seating for the visiting family and the option of a wall-mounted fold-down bed or foldout chairbed, particularly in any family space within the patient room. When selecting furniture, designers should consider such epidemiological concerns as the rising incidence of obesity. Any unnecessary sources of visual stimulation should be minimized. Wall furnishings should not be of bold patterns or colors that can be misperceived as threatening objects (such as bugs, animals, figures, etc.) by patients and/or their families. Wall coverings and colors should have a soothing, relaxing effect on the ICU population in general.

If possible, each patient room should include a well-defined family area. In a family space within the patient room, families are able to be present for shift change report, teaching sessions, care-planning discussions, and for participating in daily medical rounds. A family space within patient room provides families a more comfortable environment for activities. In this regard, consideration needs to be given to reducing environmental factors that disturb family members who remain in the patient room. Quiet, dimly lit areas for naps and sleeping can be provided along with comfortable furniture. Being able to sleep in the patient room also provides social support and reassurance for both the patient and family members.

\section{BARRIERS TO IMPLEMENTING HEALING DESIGN IN ICUS}

While much of the thrust for healing design in ICU and healthcare architecture comes from ED research literature, a lack of research in many areas concerning patients and families has been a significant barrier to healing design. For example, regarding human senses, studies on the role of tactile qualities of architecture in the process of healing has not been reported in the ED research literature. There is enough evidence available on noise/sound, light, and smell/aroma to help guide the process of healing design, but there is almost none available on tactility. This is an important limitation concerning healing design. It is suggested in the literature that touch is "the mother of all senses" [141]. In fact, haptic experience may be particularly relevant for families in ICUs, because humans seem to prefer touch in the most intense moments of their lives [142]. 
Regarding human experience, there is also a lack of evidence on the effects of design on memory, spirituality, and other deeper qualities of human nature in healthcare settings. Humans seek refuge in memory and spirituality when faced with a lifethreatening event. It is known that nature can provide spirituality and evoke memories (Table 2). However, no systematic research has yet been undertaken to study how architectural features of the ICU environment might evoke spirituality and/or memories to enhance the healing process. This is despite the fact that architecture has always been concerned with symbolism, spirituality, and memory.

The paradigm of ED research practice itself has stymied research in many areas concerning healing design in ICUs. ED researchers in general conceive the environment as a collection of attributes. For them, ED generally includes both ambient environmental features and physical design features. Ambient environmental features include noise, music, lighting (both natural and artificial), ambient temperature, and air quality, whereas physical design features include the layout of spaces, functions, furniture, fixtures and equipment, finish/surface materials, color, artwork, natural views, and environmental graphics. In numerous reported studies, researchers focus on some particular elements of the environment while controlling for the others-either statistically or through research design. There is a lack of studies reporting the interacting effects of physiological, psychological, and socio-cultural aspects of an environment on health outcomes. In the absence of any such evidence, the synthesis of research evidence in design becomes arbitrary.

Another issue that has stymied research in healing design is that hospitals and researchers alike generally have been interested in a disease-or patient-focused care emphasizing more on the clinical dimensions of ED in healthcare, particularly in ICU. Consequently, it has been difficult to translate the results of family-centered studies into health-care improvements programs within ICU. Additionally, the bulk of the studies in the ED area pertain to patient (not family) outcomes, even though in many cases these outcomes may be intertwined. Thus, further studies are needed to validate that research findings related to ED for patients are generalizable to family outcomes. Yet another important issue is that hospital administrators need to be able to prioritize those dimensions of healthcare that predict hospital outcomes better. Traditionally, empirical studies on family integration have been less successful in this regard.

Expenses are also a barrier to the healing design. It has been difficult to justify any amount of investment on the healing design in terms of hospital earnings. As a result, designers have been unwilling to apply healing design strategies. For example, gardens are a useful component of the healing design. They are probably most effective in congested urban conditions, where families often have no access to nature. However, in big cities, hospitals are built on expensive real estate. It is not easy to convince the administrators that gardens can be as cost effective as, for example, ten additional patient rooms or one additional diagnostic lab in terms of hospital earnings. It may take some time and effort before these administrators realize that creating a healing environment is not contradictory to focusing on containing cost and improving care efficiency. Studies have shown that healthcare designs based on good research evidence are solid economic investments for improving health and organizational outcomes 
[143-145]. In other words, a healing environment may help further the performance of an organization that values compassionate caring involving patients and their families.

Concerning environmental technology, some healing design recommendations are expensive while the others are not. For example, lighting systems that simulate natural light or that work with natural light, or multiple lighting systems that meet different visual, emotional and biological needs of patient families can be expensive and difficult to maintain. Similarly, ICU design that allows patient families to control lighting, music, temperature, air velocity, and/or privacy can be expensive because of the degree of controllability and flexibility required to meet the peculiar needs of every patient family. In contrast, signs to help wayfinding, HEPA filters to improve air quality, aroma to conceal unpleasant smells, or soothing color to promote calm and rest are relatively inexpensive design interventions.

More and better information related to environmental technology and cost effectiveness as related to healing outcomes is needed. Many hospitals today are unable to find creative ways to engineer the current structure for healing without huge costs. There is not enough information available to guide the selection of furniture, lighting sources and placement, surface materials, and noise abatement strategies for healing design in ICUs. This type of structural, engineering information would be extremely helpful for the engineers and architects in designing a healing environment.

Finally, a healing design in ICUs often requires cultural changes that are difficult to make. For example, recommendations are made to include family spaces within ICUs and patient rooms to improve families' access to patients and care providers. However, it can be difficult for some providers to have families around them when they serve patients. Interventional programs to change the culture of practice may be helpful in this regard. However, such programs require longer commitments, thus can become expensive.

\section{CONCLUSION}

A "healing environment of care" model for patient family in ICUs is proposed. By integrating environmental design as an important dimension, the model aims at overcoming the limitations of those family integration models that focus only on changing the culture of care. It is argued that without appropriate environmental design to support family presence and integration, any mandate to change the culture of care can increase staff stress, and hence may soon become counter-productive. It is also argued that supporting family integration through healing design involves an understanding of the physiological, psychological, as well as socio-cultural wellbeing of patient families gained through studies of their needs, experience, and behavioral responses in ICUs. Based on the existing ED research evidence, generic design recommendations are made to promote healing design in ICUs in relation to various environmental, behavioral, social, and psychosocial issues. Specific design recommendations are also made to promote healing design for family spaces in ICUs. Due to a lack of ED research on ICUs, these specific recommendations reflect mostly the author's point of view. Together, these ED recommendations may help transform the aversive, technology heavy ICU environment into a healing environment for patient families. 


\section{ACKNOWLEDGEMENT}

This work was partly supported by the University of Kansas General Research Fund allocation \#2301309. I would like to thank all the reviewers who had commented on the earlier versions of the paper.

\section{REFERENCES}

[1] Consensus Project for Quality Palliative Care. National consensus project for quality palliative care: Clinical practice guidelines for quality palliative care, executive summary. J Palliat Med, 2004, 7(5), 611-627.

[2] Bozett, F.W. and Gibbons, R., The nursing management of families in the critical care setting, Crit Care Update, 1983, 10(2), 22-7.

[3] Byers, J.F., Family discussion groups, a way to meet the needs of the ICU patient's family, Crit Care Nurse, 1983, 3(1), 16,20.

[4] Hodovanic, B.H., Reardon, D., Reese, W. and Hedges B., Family crisis intervention program in the medical intensive care unit, Heart \& Lung, 1984, 13, 243-9.

[5] Molter, N.C., Needs of relatives of critically ill patients: a descriptive study, Heart \& Lung, 1979, 8, $332-9$.

[6] Simpson, T., The family as a source of support for the critically ill adult, AACN Clin Issues Crit Care Nurs, 1991, 2, 229-35.

[7] Caplan, G., The family as a support system, in: McCubbin, H., Cauble, A., and Patterson, J., eds., Family stress, coping, and social support, Charles C. Thomas, Springfield, IL, 1982, 200-20.

[8] Elsayed, S., Greenland, B., Joyner, B., et al., Getting on board: training activities to promote the practice of family-centered care, 2nd ed., Association for the Care of Children's Health, Bethesda, MD, 1995.

[9] Institute of Medicine, Crossing the Quality Chasm: A New Health System for the 21 $1^{\text {st }}$ Century, National Academies Press, Washington, DC, 2001.

[10] Lewin, S.A., Skea, Z.C., Entwistle, V., et al., Interventions for providers to promote a patient- centered approach in clinical consultations, Cochrane Database Syst Rev, 2001, 4, CD003267.

[11] Pollack, M.M. and Koch, M.A., Association of outcomes with organizational characteristics of neonatal intensive care units, Crit Care Med, 2003, 31, 1620-1629.

[12] Roter, D.L., Hall, J.A., Kern, D.E., et al., Improving physicians' interviewing skills and reducing patients' emotional distress: A randomized clinical trial, Arch Intern Med, 1995, 155, 1877-1884.

[13] Stewart, M., Brown, J.B., Donner, A., et al., The impact of patient-centered care on outcomes, J Fam Pract, 2000, 49, 796-804.

[14] Benbassat, J., Pilpel, D. and Tidhar, M., Patients' preferences for participation in clinical decision making: A review of published surveys, Behav Med, 1998, 24, 81-88.

[15] Frosch, D.L. and Kaplan, R.M., Shared decision making in clinical medicine: Past research and future directions, Am J Prev Med, 1999, 17, 285-294.

[16] Gerteis, M., Edgman-Levitan, S., Walker, J.D. et al., What patients really want, Health Manage $Q$ 1993, 15, 2-6.

[17] Heyland, D.K., Rocker, G.M., Dodek, P.M., et al., Family satisfaction with care in the intensive care unit: Results of a multiple center study, Crit Care Med, 2002, 30, 1413-1418.

[18] The SUPPORT Principal Investigators, A controlled trial to improve care for seriously ill hospitalized patients: The study to understand prognoses and preferences for outcomes and risks of treatments (SUPPORT), JAMA, 1995, 274, 1591-1598.

[19] Molter, N., Creating healing environment for critical care, Crit Care Nurs Clin N Am, 2003, 15, 295-304. 
[20] Kutash, M. and Northrop, L., Family members' experiences of the intensive care unit waiting room, Journal of Advanced Nursing, 2007, 60(4), 384-388.

[21] Bournes, D.A. and Mitchell, G.J., Waiting: the experience of persons in a critical care waiting room, Research in Nursing \& Health, 2002, 25(1), 58-67.

[22] Davidson, J.E., Powers, K., Hedayat, K.M., Tiesze, M., Kon, A.A., Shepard, E., et al., Clinical practice guidelines for support of the family in the patient-centered intensive care unit: American College of Critical Care Medicine Task Force 2004-2005, Crit Care Med, 2007, 35(2), 605-622.

[23] Felgen, J., A caring and healing environment, Nurs Amin Q, 2004, 28(4), 288-301.

[24] Fontaine, D. K., Briggs, L. P. and Pope-Smith, B., Designing humanistic critical care environments, Crit Care Nurs Q, 2001, 24(3), 21-34.

[25] Rubert, R., Long, L. D. and Hutchinson, M. L., Creating a healing environment in the ICU, in: Roberta Kaplow, R., and Hardin, S. R., eds., Critical Care Nursing: Synergy for Optimal Outcomes, Jones \& Bartlett Publishers, 2007, pp. 27-39.

[26] Stichler, J.F., Creating healing environments in critical care units, $C C N Q, 2001,24(3), 1-20$.

[27] Rashid, M., A Decade of Adult Intensive Care Unit Design: A Study of the Physical Design Features of the Best-Practice Examples, Critical Care Nursing Quarterly, 2006, 29(4), 282-311.

[28] Baker, C.F., Sensory overload and noise in the ICU: Sources of environmental stress, CCQ, 1984, 6, 66-80.

[29] Bedsworth, J. and Molen, M., Psychological stress in spouses of patients with myocardial infarction, Heart \& Lung, 1982, 1(5), 450-456.

[30] Bouman, C., Identilying priority concerns of families of ICU patients, Dimensions of Critical Care Nursing, 1984, 3(5), 313-319.

[31] Caplan, M. and Sexton, D., Stresses experienced by spouses of patients in a coronary care unit with myocardial infarction, Focus Crit Care, 1988, 15(5), 31-40.

[32] Daley, L., The perceived immediate needs of families with relatives in the intensive care setting, Heart \& Lung, 1984, 13(3), 231-7.

[33] Forrester , D.A., Murphy, P.A., Price, D.M. and Monaghan, J.F., Critical care family needs: nursefamily member confederate pairs, Heart \& Lung, 1990, 66, 655-661.

[34] Freichels, T.A., Needs of family members of patients in the intensive care unit over time, Crit Care Nurs Q, 1991, 14(3), 16-29.

[35] Kleinpell, R.M. and Powers. M.J., Needs of family members of intensive care unit patients, Appl Nurs Res, 1992, 5(1), 2-8.

[36] Molter, N.C., and Leske, J.S. Critical care family needs inventory (CCFNI). University of Wisconsin - Milwaukee, Milwaukee, 1983. (Available from authors)

[37] Leske, J. S., Internal psychometric properties of the Critical Care Family Needs Inventory, Heart Lung, 1991, 20, 236-244.

[38] Lynn-McHale, D.J. and Bellinger, A., Need satisfaction levels of family members of critical care patients and accuracy of nurses' perceptions, Heart \& Lung, 1988, 17, 447-53.

[39] Mathis, M., Personal needs of family members of critically ill patients with and without acute brain injury, Journal of Neurosurgical Nursing, 1984, 16(1), 36-44.

[40] Norris, L. and Grove, S., Investigation of selected psychosocial needs of family members of critically ill adult patients, Heart \& Lung, 1986, 15, 194-199.

[41] Price, D.M., Forrester, D.A., Murphy, P.A. and Monaghan, J.F., Critical care family needs in an urban teaching medical center, Heart \& Lung, 1991, 20(2), 183-88.

[42] Rodgers, C.D., Needs of relatives of cardiac surgery patients during the critical care phase, Focus Crit Care, 1983, 10(5), 50-5.

[43] Titler, M.G., Bombei, C. and Schuttle, D.L., Developing family-focused care, Critical Care Nursing Clinics of North America, 1995, 7(2), 375-386. 
[44] Halm, M.A., Support and reassurance needs: Strategies for practice, Critical Care Nursing Clinics of North America, 1990, 5(1), 34-42.

[45] Titler, M.G. and Walsh, S.M., Visiting critically ill adults: Strategies for practice, Critical Care Nursing Clinics of North America, 1992, 4, 623-632.

[46] Koller, P., Family needs and coping strategies during illness crisis, Clin Issues Crit Care Nurs, 1991, $2,338-45$.

[47] Kleiber. C., Halm, M., Titler, M., et al., Emotional responses of family members during a critical care hospitalization, Am J Crit Care, 1994, 3(1), 70-76.

[48] Halm, M. and Titler, M., Appropriateness of critical care visitation: Perceptions of patient, family members, nurses and physicians, J Nurs Quality Assurance, 1990, 5(1), 25-37.

[49] Halm, M.A., Titler, M.G., Kleiber, C., et al., Behavioral responses of family members during critical illness, Clin Nurs Res, 1993, 2, 414-437.

[50] Irwin, B.L. and Bleier, J.R., Supportive measures for the relatives of the fatally ill, Commun Nurs Res, 1973, 6, 126.

[51] Halm, M.A., Effects of support groups on anxiety of family members during critical illness, Heart \& Lung, 1990, 19, 62-71.

[52] Perez-San Gregorio, M.A., Blanco-Picabia, A., Murillo-Cabezas, F., Dominguez-Roldan, J.M., Sanchez, B. and Nunez-Roldan, A., Psychological problems in the family members of gravely traumatized patients admitted into an intensive care unit, Intensive Care Med, 1992, 18, 278-281.

[53] Pochard, F., Azoulay, E., Chevret, S., et al., Symptoms of anxiety and depression in family members of intensive care unit patients: Ethical hypothesis regarding decision-making capacity, Crit Care Med, 2001, 29, 1893-1897.

[54] Titler, M., Cohen, M. and Craft, M., Impact of critical illness event on the family: Perceptions of patients, spouses, children and nurses, Heart \& Lung, 1991, 20, 174-182.

[55] Walters, A.J., A hermeneutic study of the experiences of relatives of critically ill patients, J Adv Nurs, $1995,22,998-1005$.

[56] Walters, A.J., The lifeworld of relatives of critically ill patients: a phenomenological hermeneutic study, Int J Nurs Pract, 1995, 1, 18-25.

[57] Jamerson, P.A., Scheibmeir, M., Bott, M.J., Crighton, F., Hinton, R.H. and Cobb, A.K., The experiences of families with a relative in the intensive care unit, Heart \& Lung, 1996, 25(4), 467-474.

[58] Burr, G., Contextualizing critical care family needs through triangulation: an Australian Study, Intensive \& Critical Care Nursing, 1998, 14(4), 161-169.

[59] Auerbach, S.M., Kiesler, D.J., Wartella, J., et al., Optimism, satisfaction with needs met, interpersonal perceptions of the healthcare team, and emotional distress in patients' family members during critical care hospitalization, American Journal of Critical Care, 2005, 14(3), 202-210.

[60] Gerteis, M., Edgman-Levitan, S., Daley, J., Delbanco, T.L., eds. Through the Patient's Eyes: Understanding and Promoting Patient-Centered Care, Jossey-Bass, San Francisco, CA, 1993.

[61] Dowling, J., Vender, J., Guilianelli, S. and Wang, B., A Model of Family-Centered Care and Satisfaction Predictors, Chest, 2005, 128, 81S-92S.

[62] Arthur, P. and Passini, R., Wayfinding: People, signs, and architecture, McGraw-Hill Book Co., New York, 1992.

[63] Bosma, K.J. and Ranieri, V.M., Filtering out the noise: evaluating the impact of noise and sound reduction strategies on sleep quality for ICU patients, Crit Care, 2009, 13(3), 151. Published online 2009 May 29. doi: 10.1186/cc7798.

[64] Brown, B., Wright, H. and Brown, C., A post-occupancy evaluation of wayfinding in a pediatric hospital: Research findings and implications for instruction, Journal of Architectural \& Planning Research, 1997, 14(1), 35-51.

[65] Corr, M., Reducing occupational stress in intensive care, Nursing in Critical Care, 2000, 5(2), 76-81. 
[66] Carpman, J.R. and Grant, M.A., Design that cares: Planning health facilities for patients and visitors, 2nd edn., American Hospital Publishing, Inc., Chicago, 1993.

[67] Cooke, B. and Ernst, E., Aromatherapy: A systematic review, British Journal of General Practice, 2000, 50, 493-496.

[68] Daykin, N. and Bunt, L., Music as a Resource for Health and Well-Being, in: Ingrid Söderback, ed., International Handbook of Occupational Therapy Interventions, Springer, New York, 2009, 453-46.

[69] Golden, R.N., Gaynes, B.N., Ekstrom, R.D., Hamer, R.M., Jacobsen, F.M., Suppes, T., et al., The efficacy of light therapy in the treatment of mood disorders: A review and meta-analysis of the evidence, American Journal of Psychiatry, 2005, 162(4), 656-662.

[70] Handzo, G., and Wilson, J.C., Spirituality - inner resources for healing, in: Charmel, P., Frampton, S.B., Gilpin, L., eds., Putting Patients First-Designing and Practicing Patient-Centered Care, Jossey-Bass, San Francisco, 2003, 89-104.

[71] Hanser, S. B., From Ancient to Integrative Medicine: Models for Music Therapy, Music and Medicine, 2009, 1(2), 87-96.

[72] Joye, Y., Architectural lessons from environmental psychology: the case of biophilic architecture, Review of General Psychology, 2007, 11(4), 305-328.

[73] Marcus, C.C. and Barnes, M., Gardens in healthcare facilities: Uses, therapeutic benefits, and design recommendations, The Center for Health Design, Concord, CA, 1995.

[74] Marcus, C.C. and Barnes, M., eds., Healing gardens: Therapeutic benefits and design recommendations, Wiley, New York, 1999.

[75] Marberry, S.O. and Zagon, L., The Power of Color: Creating Healthy Interior Color, John Wiley \& Sons, Inc, New York, 1995.

[76] O’Brien, M.E., Spirituality in Nursing, Jones and Bartlett, Sudbury, CT, 2003.

[77] Rashid, M., Face-to-Face Interaction among Clinicians and Hospital Design: A Theoretical Model, Health Environments Research \& Design Journal, 2009, 2(3), 62-84.

[78] Rashid, M. and Zimring, C., A Review of the Empirical Literature on the Relationships between Indoor Environment and Stress in Healthcare and Office Settings: Problems and Prospects of Sharing Evidence, Environment and Behavior, 2008, 40(3), 151-190.

[79] Robins, J.L.W., The science and art of aromatherapy, Journal of Holistic Nursing, 1999, 17, 5-17.

[80] Schweitzer, M., Gilpin, L. and Frampton, S., Healing spaces: elements of environmental design that make an impact on health, The Journal of Alternative and Complementary Medicine, 2004, 10, S1, s71-s83.

[81] Townsend, M., Kladder, V., Ayele, H. and Mulligan, T., Systematic review of clinical trials examining the effects of religion on health, Southern Med J, 2002, 95, 1429-1424.

[82] Ulrich, R.S., Biophilic theory and research for health design, in: S. Kellert, J. Heerwagen, \& M. Mador, eds., Biophilic design: Theory, science and practice of bringing buildings to life, John Wiley, New York, 2008.

[83] Ulrich, R.S., Effects of gardens on health outcomes: Theory and research, in: Marcus, C.C., \& Barnes, M., eds., Healing Gardens, Wiley, New York, 1999, 27-86.

[84] Ulrich, R., Zimring, C., Quan, X., Joseph, A. and Choudhary, R., The role of the physical environment in the hospital of the 21st century: A once-in-a-lifetime opportunity, The Center for Health Design, Concord, CA, 2004.

[85] Ulrich, R.S., Zimring, C., Zhu, X., et al., A review of the literature on evidence-based healthcare design, Healthcare Environment Research and Design Journal, 2008, 1(3), 61-125.

[86] Ulrich, R. S., Zimring, C., Quan, X. and Joseph, A., The environment's impact on stress, in: Marberry, S., ed., Improving healthcare with better building design, Health Administration Press, Chicago, 2006, 37-61.

[87] White, J.M., State of the science of music interventions, Crit Care Clin, 2000, 12, 219-225.

[88] Xie, H., Kang, J. and Mills, G. H., Clinical review: The impact of noise on patients' sleep and the effectiveness of noise reduction strategies in intensive care units, Crit Care, 2009, 13(2), 208. 
[89] Young, C., Spiritually healing environments, in: Spirituality, Health, and Healing, Jones \& Bartlett Publishers, 2006, 147-168.

[90] Blomkvist, V., Eriksen, C.A., Theorell, T., Ulrich, R.S. and Rasmanis, G., Acoustics and psychosocial environment in intensive coronary care, Occupational and Environmental Medicine, 2005, 62, $132-139$.

[91] Cmiel, C.A., Karr, D.M., Gasser, D.M., Oliphant, L.M. and Neveau, A.J., Noise control: A nursing team's approach to sleep promotion, American Journal of Nursing, 2004, 104, 40-48.

[92] Johnson, A.N., Neonatal response to control of noise inside the incubator, Pediatr Nurs, 2001, 27, $600-605$.

[93] Johnson, A.N., Adapting the neonatal intensive care environment to decrease noise, J Perinat Neonatal Nurs, 2003, 17, 280-288.

[94] Gast, P.L. and Baker, C.F., The CCU patient: Anxiety and annoyance to noise, Crit Care Nurs Q, 1989, $12,39-54$.

[95] Baker, C.F., Discomfort to environmental noise: Heart rate responses of SICU patients, Crit Care Nurs $Q, 1992,15,75-90$.

[96] Baker, C.F., Annoyance to ICU noise: A model of patient discomfort, Crit Care Nurs Q, 1993, 16, 83-90.

[97] Baker, M.A., and Holding, M.A., The effects of noise and speech on cognitive task performance, J Gen Psychol, 1993, 120, 339-355.

[98] Balogh, D., Kittinger, E., Benzer, A., et al., Noise in the ICU, Intensive Care Med, 1993, 19, 343-346.

[99] Falk, S.A. and Woods, N.F., Hospital noise-levels and potential health hazards, New Engl J Med, 1973, $289,774-781$.

[100] Freedman, N.S., Kotzer, N. and Schwab, R.J., Patient perception of sleep quality and etiology of sleep disruption in the intensive care unit, Am J Respir Crit Care Med, 1999, 159, 1155-1162.

[101] Grumet, G., Pandemonium in the modern hospital, NEMJ, 1993, 328, 433-437.

[102] Chlan, L.L., Effectiveness of a music therapy intervention on relaxation and anxiety for patients receiving ventilator assistance, Heart \& Lung, 2000, 27, 169-176.

[103] Updike, P., Music therapy results for ICU patients, DCCN, 1990, 9, 39-45.

[104] Bolwerk, C.A., Effects of relaxing music on state anxiety in myocardial infarction patients, Crit Care Nurs $Q, 1990,13,63-72$.

[105] White, J.M., Effects of relaxing music on cardiac autonomic balance and anxiety after acute myocardial infarction, Am J Crit Care, 1999, 8, 220-230.

[106] Zimmerman, L., Nieveen, J., Barnason, S. and Schmaderer, M., The effects of music interventions on postoperative pain and sleep in coronary artery bypass graft (CABG) patients, Sch Inq Nurs Pract, 1996, 10, 2, 153-170.

[107] Byers, J.F., and Smyth, K.A., Effect of a music intervention on noise annoyance, heart rate, and blood pressure in cardiac surgery patients, Am J Crit Care, 1997, 6, 183-191.

[108] Davidson, J.E., Boyer, M.L., Casey, D., Matzel, S.C. and Walden, C.D., Gap analysis of cultural and religious needs of hospitalized patients, Crit Care Nurs Q, 2008, 31(2), 119-126.

[109] Steptoe, A. and Appels, A., Eds., Stress, Personal Control, and Health, Chichester, John Wiley, England, 1989.

[110] Evans, G. W. and Cohen, S., Environmental Stress, in: Stokols, D. and Altman, I., eds., Handbook of Environmental Psychology, 2 vols., John Wiley, New York, 1987, pp. 571-610.

[111] Winkel, G. H. and Holahan, C. J., The environmental psychology of the hospital: is the cure worse than the illness?, Prevention in Human Services, 1986, 4, 11-33.

[112] Dubbs, D., Privacy please, Health Facil Manage, 2003, 16, $20-24$.

[113] Gordin, P. and Johnson, B.H., Technology and family-centered perinatal care: Conflict or synergy, JOGNN, 1999, 28, 401-408. 
[114] Cohen, S. and Syme, S. L., eds., Social Support and Health, Academic Press, New York, 1985.

[115] Saranson, I. G., and Saranson, B. R., eds., Social Support: Theory, Practice, and Applications, Nijhoff, The Hague, 1985.

[116] Laitinen, P. and Isola, A., Promoting participation of informal caregivers in the hospital care of the elderly patient: Informal caregivers' perceptions, Journal of Advanced Nursing, 1996, 23(5), 942-947.

[117] Rashid, M., Face-to-Face Interaction among Clinicians and Hospital Design: A Theoretical Model, Health Environments Research \& Design Journal, 2009, 2(3), 62-84.

[118] Chaudhury, H., Mahmood, A. and Valente, M., Advantages and disadvantages of single versus multiple-occupancy rooms in acute care environments-A review and analysis of the literature, Environment and Behavior, 2005, 37(6), 760-786.

[119] Sallstrom, C., Sandman, P.O. and Norberg, A., Relatives' experience of the terminal care of long-term geriatric patients in open-plan rooms, Scandinavian Journal of Caring Science, 1987, 1(3-4), 133-140.

[120] Gurasio-Howard, L. and Malloch, K., Centralized and decentralized nurse station design: an examination of caregiver communication, work activities, and technology, Health Environment Research \& Design Journal, 2007, 1(1), 44-57.

[121] Melin, L. and Gotestam, K.G., The effects of rearranging ward routines on communication and eating behaviors of psychogeriatric patients, Journal of Applied Behavior Analysis, 1981, 14(1), 47-51.

[122] Peterson, R., Knapp, T. and Rosen, J., The effects of furniture arrangement on the behavior of geriatric patients, Behavior Therapy, 1977, 8, 464-467.

[123] Holahan, C., Seating patterns and patient behavior in an experimental dayroom, Journal of Abnormal Psychology, 1972, 80(2), 115-124.

[124] Sommer, R. and Ross, H., Social interaction on a geriatrics ward, The International Journal of Social Psychiatry, 1958, 4(2), 128-133.

[125] Astedt-Kurki, P., Paavilainen, E., Tammentie, T. and Paunonen-Ilmonen, M., Interaction between adult patients' family members and nursing staff on a hospital ward, Scandinavian Journal of Caring Sciences, 2001, 15(2), 142-150.

[126] Miwa, Y. and Hanyu, K., The effects of interior design on communication and impressions of a counselor in a counseling room, Environment and Behavior, 2006, 38(4), 484-502.

[127] Harris, D., Environmental quality and healing environments: A study of flooring materials in a healthcare telemetry unit, Doctoral dissertation, Texas A\&M University, College Station, 2000.

[128] Zimring, C., The Costs of Confusion: Non-Monetary and Monetary Costs of Emory University Hospital Wayfinding System, Georgia Institute of Technology, Atlanta, 1990.

[129] Haq, S. and Zimring, C., Just down the road a piece: The development of topological knowledge of building layouts, Environment \& Behavior, 2003, 35(1), 132-160.

[130] Peponis, J., Zimring, C. and Choi, Y.K., Finding the building in wayfinding, Environment \& Behavior, 1990, 22(5), 555-590.

[131] Carpman, J.R., Grant, M. and Simmons, D., Wayfinding in the hospital environment: The impact of various floor numbering alternatives, Journal of Environmental Systems, 1983, 13(4), 353-364.

[132] Levine, M., Marchon, I. and Hanley, G., The placement and misplacement of you-are-here maps, Environment \& Behavior, 1984, 16(2), 139-157.

[133] Nelson-Shulman, y., Information and environmental stress: Report of a hospital intervention, Journal of Environmental Systems, (1983-84), 13(4), 303-316.

[134] Wright, P., Hull, A.J. and Lickorish, A., Navigating in a hospital outpatients' department: The merits of maps and wall signs, The Journal of Architectural \& Planning Research, 1993, 10(1), 76-89.

[135] Carpman, J. R., Grant, M. and Simmons, D., No more mazes: Research about design for wayfinding in hospitals, The University of Michigan Hospitals, Ann Arbor, Michigan, 1984. 
[136] Krakauer, E.L., Crenner, C. and Fox, K., Barriers to optimum end-of-life care for minority patients, J Am Geriatr Soc., 2002, 50(1), 182-190.

[137] Bowman, K.W. and Singer, P.A., Chinese seniors' perspectives on end-of-life decisions, Soc Sci Med. 2001, 53(4), 455-464.

[138] Kagawa-Singer, M. and Blackhall, L.J., Perspectives on care at the close of life. Negotiating crosscultural issues at the end of life: "you've got to go where he lives," JAMA, 2001, 286(23), 2993-3001.

[139] Ekblad, S., Marttila, A. and Emilsson, M., Cultural challenges in end-of-life care: reflections from focus groups' interviews with hospice staff in Stockholm, J Adv Nurs, 2000, 31(3), 623-630.

[140] Hopp, F.P. and Duffy, S.A., Racial variations in end-of-life care, J Am Geriatr Soc., 2000, 48(6), 658-663.

[141] Montagu, A., Touching: The Human Significance of the Skin, Harper \& Row, New York, 1986.

[142] Pallasmaa, J., The Eyes of the Skin: Architecture and the Senses, Wiley, 2005.

[143] Issel, M.L. and Kahn, D., The economic value of caring, Health Care Manage Rev, 1998, 23, 43-53.

[144] Malloch, K., Healing models for organizations: description, measurement, outcomes, Journal of Healthcare Management, 2000, 45, 332-45.

[145] Sadler, B., DuBose, J.R., Malone, E.B. and Zimring, C., The business case for building better hospitals through evidence-based design, The Center for Health Design, Concord, CA, 2008. 


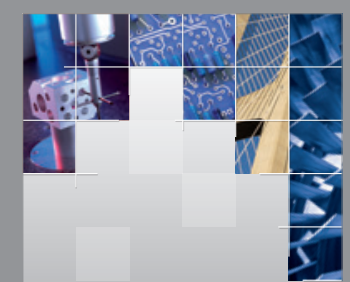

\section{Enfincering}
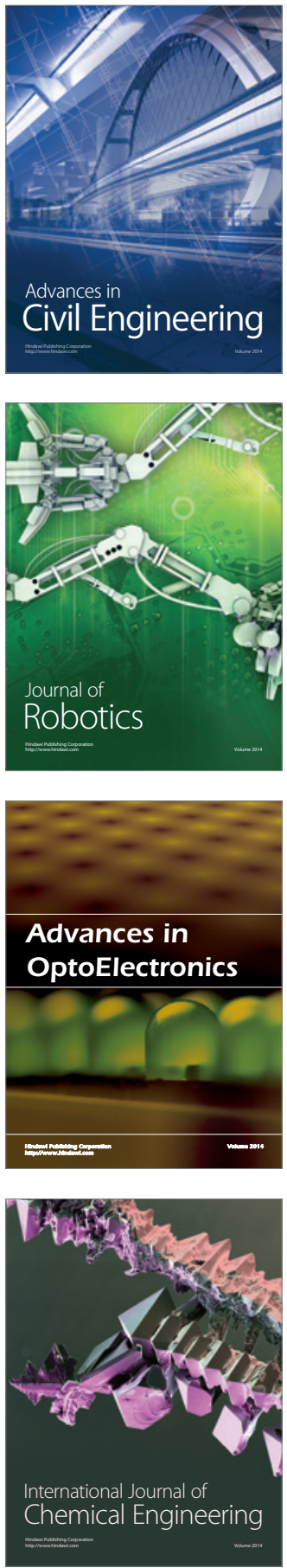

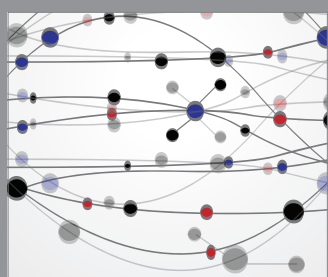

The Scientific World Journal

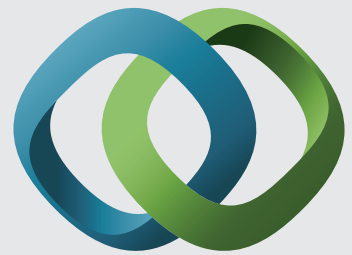

\section{Hindawi}

Submit your manuscripts at

http://www.hindawi.com
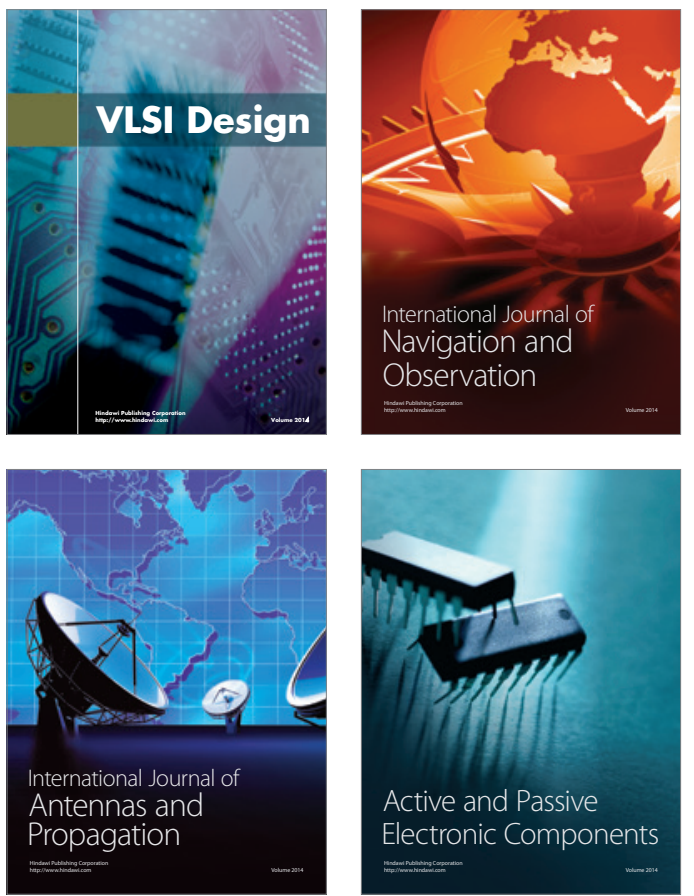
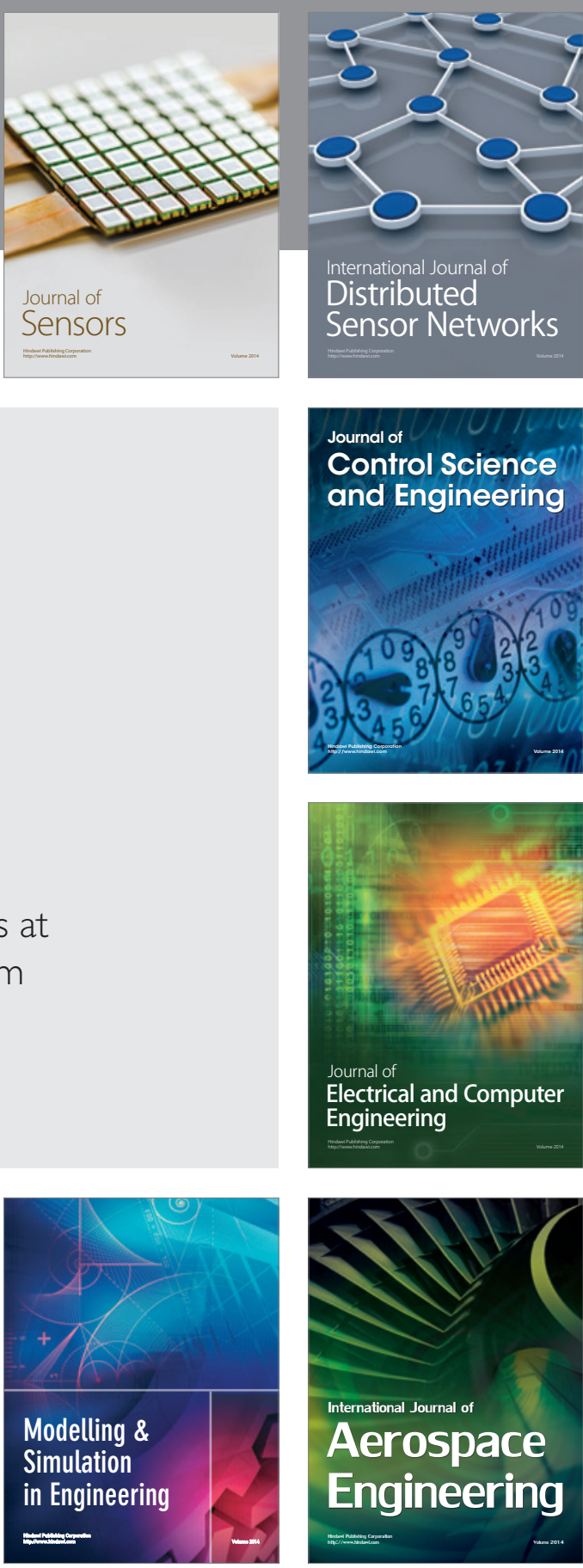

International Journal of

Distributed

Sensor Networks

Journal of

Control Science

and Engineering
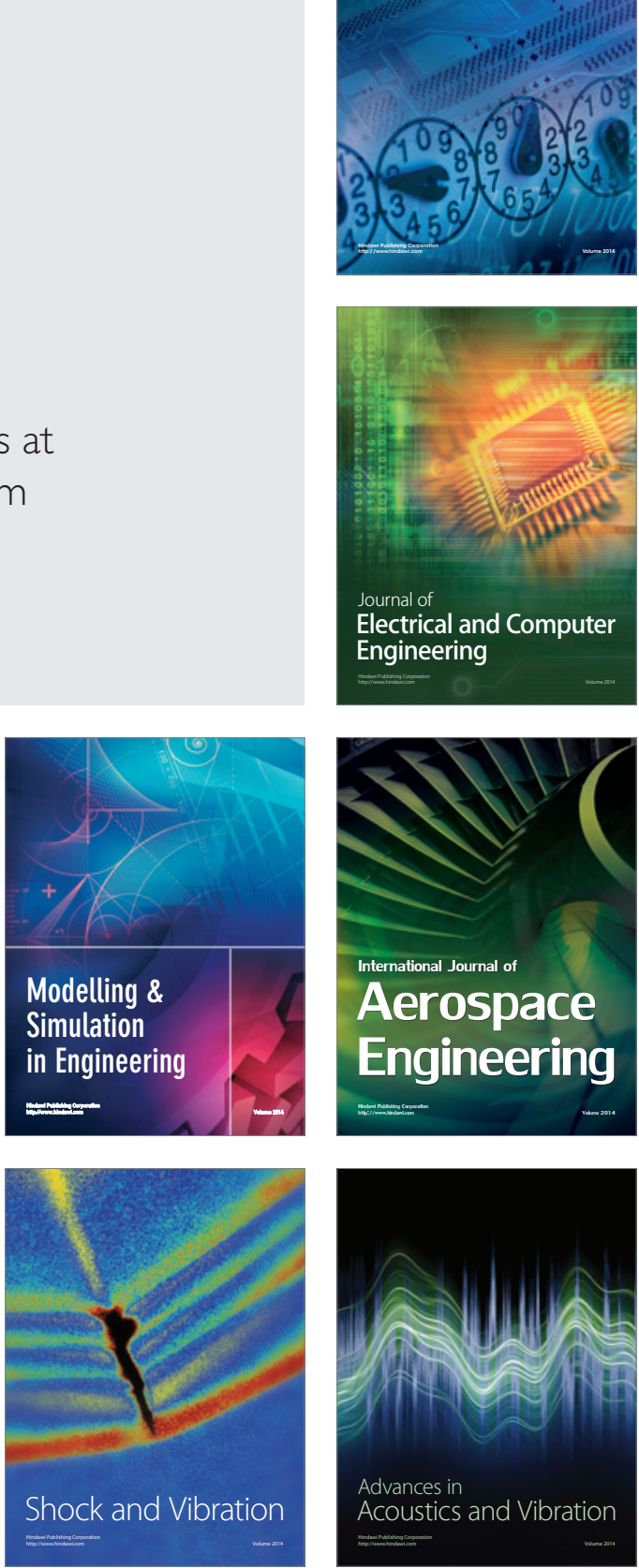